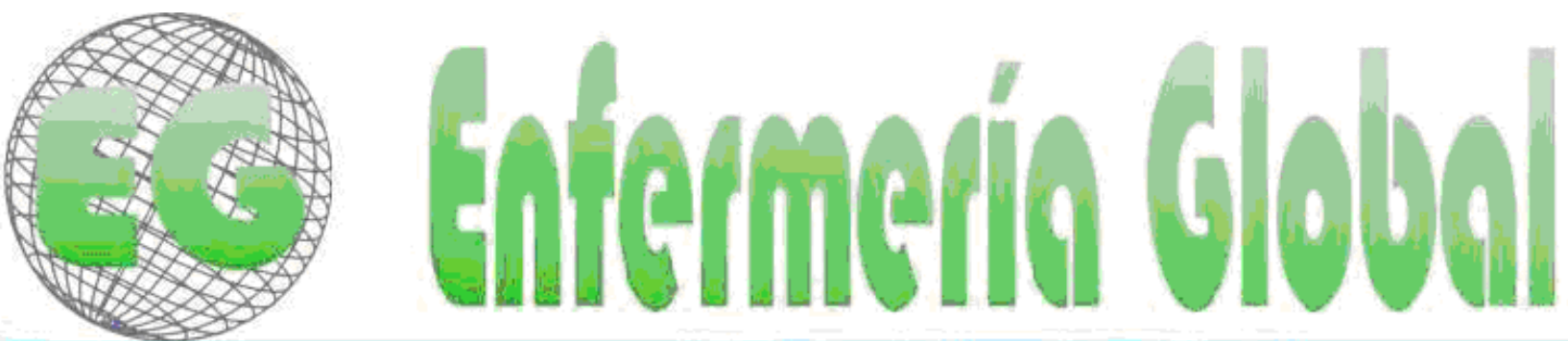

$19 \operatorname{SN} 1695-6144$

$\mathrm{N}^{\circ} 16$

Revista electrónica cuatrimestral de Enfermería

Junio 2009

www.um.es/eglobal/

REFLEXIONES - ENSAYOS

\title{
ENFERMERÍA Y EL PACIENTE EN SITUACIÓN TERMINAL
}

NURSING CARE FOR THE TERMINALLY ILL PATIENTS.

\author{
*Míguez Burgos, A., "*Muñoz Simarro, D. \\ *Enfermero. FREMAP Ciudad Jardín.**Enfermero. DCCU Distrito. Sevilla. \\ Palabras clave: DUE, Muerte, Proceso terminal, Medidas terapéuticas, Calidad de vida, Dolor.
}

Keywords: Nursing, Death, Process terminal, Terminal patients, Therapeutic action, quality of live, Pain

\section{RESUMEN}

El hecho de la muerte, de la no vida, ha sido en todas las sociedades, para todos los grupos humanos y para todos los individuos una situación que se ha afrontado de muy diversas formas. En general, a pesar de que la muerte es una parte, un fenómeno de la propia existencia, existe un rechazo hacia ella, hacia las personas que se hallan cercanas a la situación de muerte. En este artículo se presenta una reflexión sobre el proceso terminal, sus características, fases, influencia de nuestras actividades como D. U. E., etc. El objetivo de este artículo no es demostrar unas hipótesis, tampoco lo es establecer verdades absolutas e indiscutibles, ya que el tema se presta a un interminable debate donde siempre las conclusiones serán personales. Lo que pretendemos es invitar a la reflexión, personal o en grupo, para fomentar actitudes positivas en los profesionales de Enfermería de cara al cuidado de enfermos terminales. Estas actitudes se traducirán en una mejor calidad de la asistencia. Terminamos finalmente con una serie de recomendaciones prácticas para que, por medio del cambio de actitudes, mejoremos la asistencia prestada a pacientes terminales, en orden a un aumento en su calidad de vida.

\section{ABSTRACT}

The fact of death, non-life, has been in all societies, for all groups and all individuals a situation that has been faced in many different ways. In general, despite the fact that death is a part, a phenomenon of existence, there is a rejection of it, by people who are close to the situation of death. This article presents a reflection of the terminal process, its features, phases, and influence over activities such as DUE etc. The aim of this paper is not to show assumptions, nor to establish absolute indisputable truths, because the topic lends itself to an endless debate in which the conclusions are always personal. What we want is to invite reflection, personal or group counseling to foster positive attitudes in nursing care for the terminally ill patients. These attitudes will translate into better quality of care. We end with a series of practical recommendations that, by changing attitudes, we should improve care provided to terminally ill patients in order to increase their quality of life. 


\section{INTRODUCCIÓN. CONCEPTOS.}

El hombre es conocedor del proceso de vida y sabe que envejece y tiene que morir, pero no quiere envejecer ni morir. Hay que considerar que la muerte forma parte de un ciclo vital.

Las personas "conviven" con el miedo a la muerte, pero pueden posponer el plantearse el problema, que les resulta angustioso en sumo grado: así se defienden de la ansiedad que les crea. Los profesionales de Enfermería, por nuestras responsabilidades inherentes, estamos continuamente enfrentados a la realidad de la muerte de otras personas. Esta confrontación a menudo resulta dolorosa o difícil, pero debemos encontrar una actitud serena y equilibrada para aliviar nuestros sentimientos de tensión y cubrir al mismo tiempo las necesidades del paciente con enfermedad terminal.

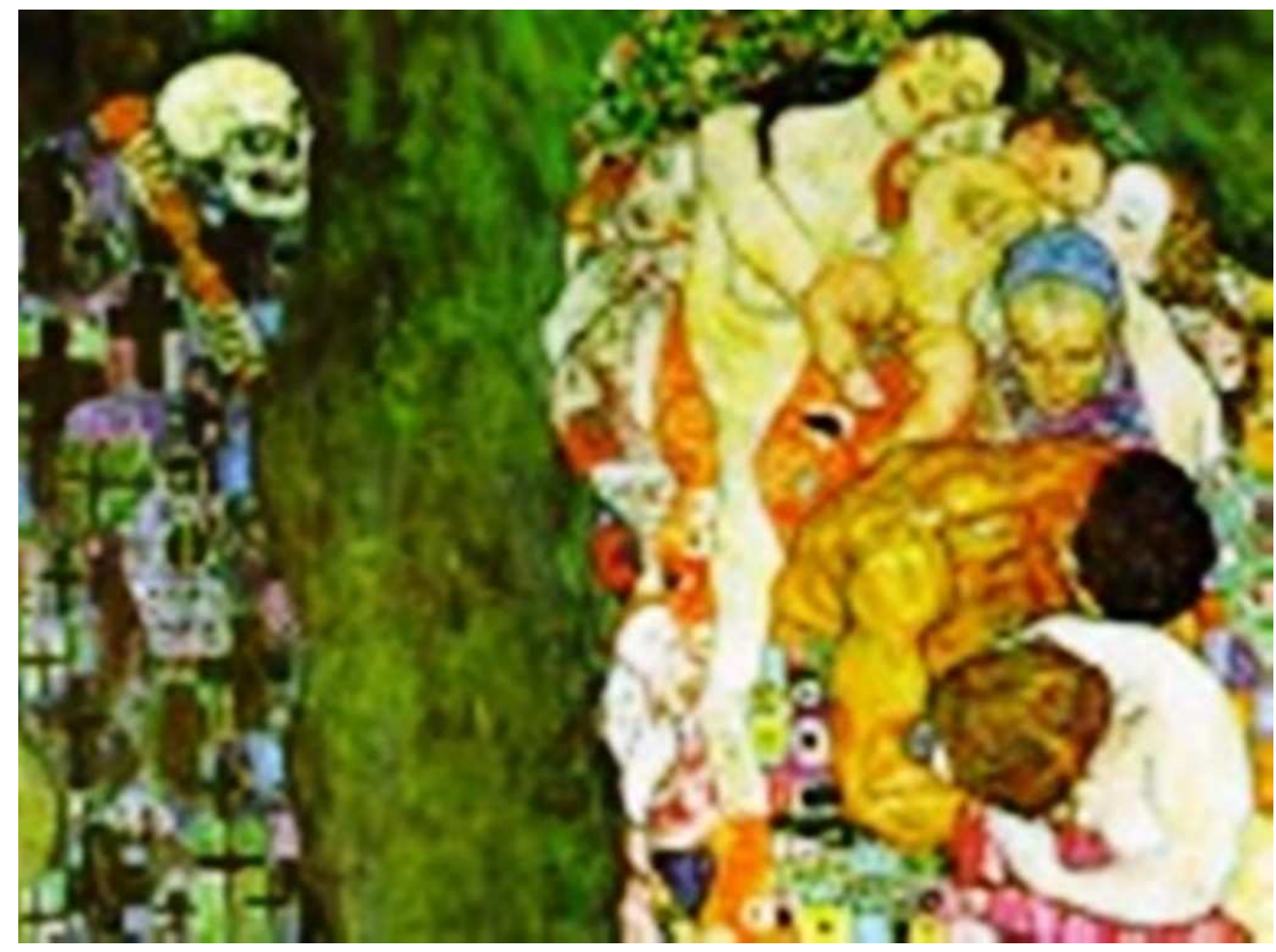

1.- El hombre siempre ha convivido con el miedo a la muerte.

Las personas, en general, deberíamos ser consciente desde un principio y aceptar como natural el proceso de la vida, el continuum salud-enfermedad, así como, el desarrollo final de esta, que es la muerte, intentando desdramatizar y eliminar tabúes al respecto. Los profesionales de enfermería no sólo tenemos que entender esto y aceptarlo, sino que debemos asumir profesionalmente que al igual que es parte de nuestro trabajo asistir, asesorar y acompañar al nacimiento de la vida, igualmente es en el fin de esta (la muerte).

Para poder llevar esta práctica de la mejor manera posible debemos ser conocedores de las peculiaridades y connotaciones que la muerte tiene en las distintas culturas, razas o etnias que se mezclan en nuestra sociedad.

Debemos tener en cuenta ante un paciente moribundo que su muerte es irremediablemente personal, al igual que la vida. La certidumbre de la muerte es lo que humaniza, y de los aspectos más importantes que debemos de considerar es que el moribundo es un ser vivo, y 
la proximidad de la muerte les hace más sensibles sabios y nobles. La asistencia al enfermo no curable pasa por la desdramatización de la muerte, ya que el principal problema en estos pacientes es aceptarla, por el problema que plantea su negación.

Hay que añadir, además, que aceptar la muerte del paciente contradice el objetivo esencial de las profesiones sanitarias, que es conservar la salud y la vida. Por ello, erróneamente, al individuo que entra en un proceso terminal se le cuida para que se cure y no para que viva con su calidad de vida el tiempo que le queda, ocultándose en un gran porcentaje de casos la realidad y negándosele la oportunidad de ser él mismo hasta el final.

Otro aspecto de vital importancia es que la presencia de la muerte remueve las actitudes del personal sanitario y, recíprocamente, esas actitudes influyen positiva o negativamente en el proceso terminal del paciente al que se está asistiendo y en sus familiares. Es decir, existe un feed-back entre el proceso terminal y las actitudes del personal sanitario. De ahí la necesidad de que nuestras actitudes, como profesionales de Enfermería ante esta realidad cotidiana, sean lo más positivas y acertadas posibles, y así contribuir con nuestros cuidados a una mejora en la calidad de vida de los enfermos terminales.

Actualmente la muerte tiende a ignorarse, se separan los que van a morir o se les tiene dormidos, hay que procurar o permitir que el moribundo sea activo ante su propia muerte. No hay que olvidar que el moribundo sigue siendo una persona viva y tiene derecho a una muerte digna: participación y autonomía en el proceso, poder elegir, respetar las decisiones del paciente, comunicación del paciente moribundo con su familia y sus médicos, resolución de asuntos personales, aceptación del proceso, no confusión mental, no dolor, etc.

Para todas las personas es necesario saber de la muerte, puesto que es una etapa vital. Sin embargo, para los profesionales sanitarios saber de la muerte es imprescindible, porque el dolor, la enfermedad, y la muerte están presentes en las situaciones de cuidados. El saber sobre la muerte no se aprende como una asignatura más, como un saber científico, ni tampoco se aplica como se aplica este último. El saber científico se transmite por el lenguaje a través del cual establecemos una relación única y unidireccional entre una palabra y una cosa. Sin embargo el saber sobre la muerte se aprende en las relaciones con otras personas. En estas relaciones descubrimos dónde y cómo se manifiestan las rupturas de comunicación entre personas, entre grupos, en la sociedad en general. Allí es donde se filtra la muerte, en los puntos donde falla el sentido compartido.

Como profesionales, tenemos la función social de encontrar explicaciones a los problemas humanos que en la vida no se encuentran. Tenemos que comprender para poder aliviar, cuidar y curar. Una formación en las relaciones interpersonales permite aprender los valores personales, sociales y culturales que influyen en nosotros mismos, y en las personas que tratamos, respecto de las actitudes adoptadas ante las pérdidas y la separación.

\section{Criterios de situación del enfermo terminal según la Sociedad Española de Cuidados Paliativos.-}

- Presencia de una enfermedad avanzada, progresiva e incurable.

- Falta de posibilidades razonables de respuesta al tratamiento específico.

- Presencia de numerosos problemas o síntomas.

- Gran impacto emocional en paciente, familia y equipo terapéutico, muy relacionado con la presencia explícita o no, de la muerte.

- Pronóstico de vida inferior a 6 meses. (1) (2) 


\section{II.- DESARROLLO DEL PROCESO TERMINAL.}

Desde el comienzo de la vida la muerte siempre ha sido desagradable, y siempre ha buscado algo más allá. Nuestro inconsciente no reconoce un verdadero final de nuestra vida aquí en la tierra, nos es inconcebible morir por una causa natural o vejez. La muerte de por sí va asociada a un acto aterrador, algo que exige pena y castigo.

Hacer frente a un paciente después de diagnosticar una enfermedad incurable siempre es difícil. La cuestión no es preguntarnos cómo deberíamos de decírselo sino cómo compartirlo. EI D. U. E. debe ser capaz de hablar francamente sin sinónimos de muerte inminente, debe de dejar una puerta abierta a la esperanza. Es fundamental que haga comprender al paciente que no está todo perdido, que no va a desahuciarle por el hecho de ser moribundo pues es una batalla que van a librar juntos paciente familia y personal sanitario sea cual sea el resultado. A la pregunta de cuánto tiempo va a vivir se le puede contestar con que nadie lo sabe puesto que de la forma contraria es una de las formas peores de tratar al enfermo. Un caso especial sería informar al jefe de familia de la brevedad de su futura vida aunque en este caso también se le puede comentar que ponga en orden sus cosas mientras tenga tiempo y fuerza para hacerlo.

En el proceso terminal se dan distintas fases (3), (4) que enumeramos a continuación:

\section{1aㅡ Fase: Negación y Aislamiento}

La mayoría al enterarse de su enfermedad mortal reaccionan diciendo, "no, yo no, no puede ser verdad ". Esta negación es común tanto en aquellos a los que se les comunica directamente desde un principio su enfermedad, y a aquellos a los que no se les decía explícitamente y que llegaban a aquella conclusión por si mismos.

La negación, por lo menos la negación parcial, es habitual en casi todos los pacientes, no sólo durante las primeras fases de la enfermedad o al enterarse del diagnóstico, sino también más adelante, de vez en cuando. La negación funciona como un amortiguador después de una noticia inesperada e impresionante, permite recobrarse al paciente y, con el tiempo, movilizar otras defensas menos radicales. 


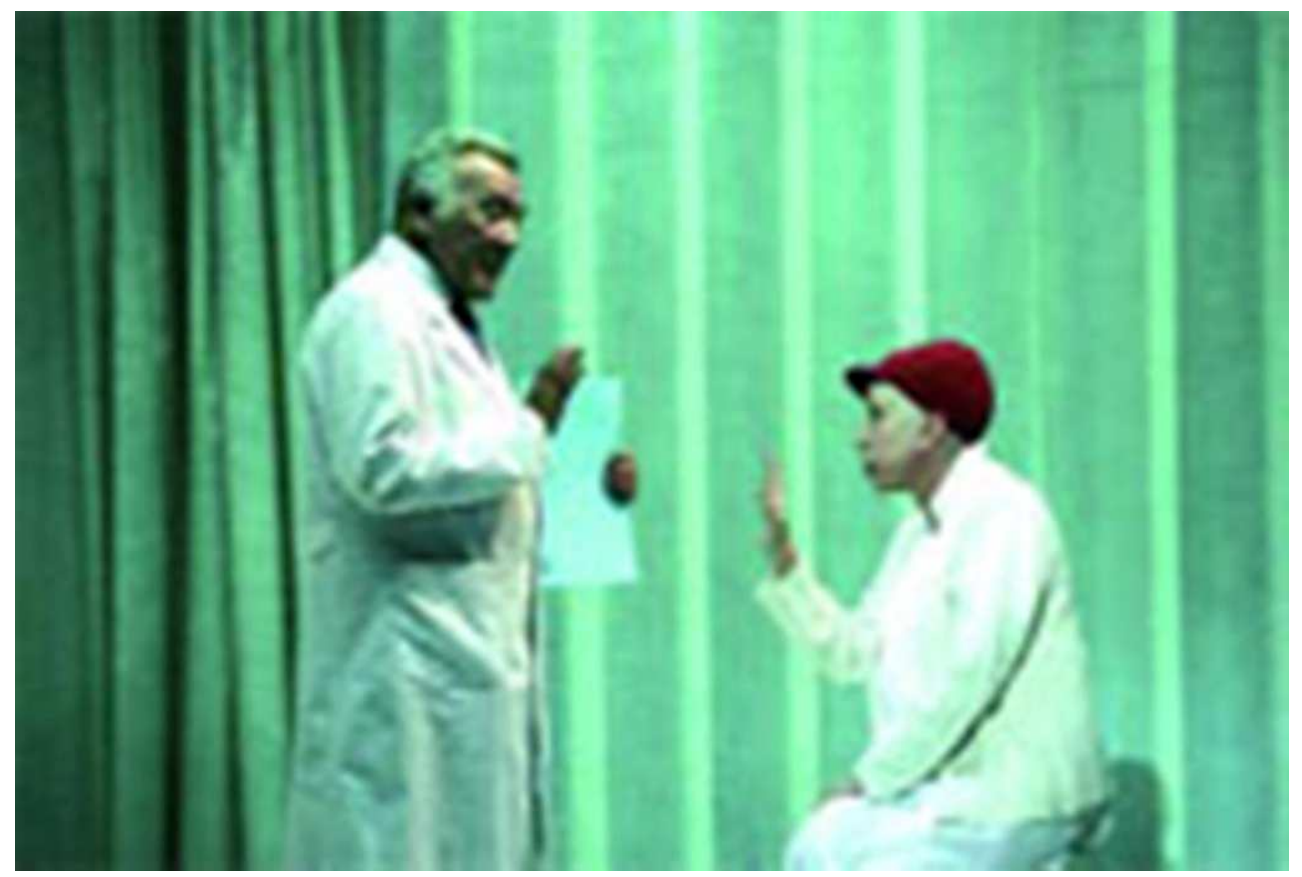

2.- 1aㅡ Fase: Negación y Aislamiento tras comunicársele su enfermedad.

La necesidad de negación existe en todos los pacientes alguna vez, luego, la necesidad va y viene, y el oyente sensible y perceptivo reconocerá esto y respetará las defensas del paciente sin hacerle consciente de sus contradicciones. Cuando estén dispuestos a hablar se abrirán, y compartirán su soledad, unas veces con palabras, otras con pequeños gestos 0 comunicaciones no orales.

\section{2aㅡ Fase: Ira}

Cuando no se puede seguir manteniendo la primera fase de negación, es sustituida por sentimientos de ira, envidia, y resentimiento. Les surge la siguiente pregunta: “¿Por qué yo?". Esta fase de ira a diferencia de la anterior es muy difícil de afrontar para al familia y el personal. Esto se debe a que la ira se desplaza en todas las direcciones y se proyecta contra lo que les rodea, a veces casi al azar.

\section{3ㅁ Fase: Pacto}

En realidad, el pacto es un intento de posponer los hechos; incluye un premio "a la buena conducta ", además fija un plazo de "vencimiento " impuesto por uno mismo y la promesa implícita de que el paciente no pedirá nada más si se le concede este aplazamiento.

\section{4믈 Fase: Depresión}

Cuando el paciente desahuciado no puede seguir negando su enfermedad, su insensibilidad o estoicismo, su ira y su rabia serán pronto sustituidas por una gran sensación de pérdida. Todas estas circunstancias y otras añadidas son razones de depresión para el paciente moribundo que va causar un dolor preparatorio por el que ha de pasar el paciente desahuciado para disponerse a salir de este mundo.

Cuando la depresión es un instrumento para prepararse a la pérdida inminente de todos los objetos de amor, entonces los ánimos y las seguridades no tienen tanto sentido para facilitar 
el estado de aceptación. Y si se les permite expresar su dolor en este tipo de depresión, encontrará mucho más fácil la aceptación final.

En el dolor preparatorio no se necesitan palabras o se necesitan muy pocas. Es mucho más un sentimiento que puede expresarse mutuamente y a menudo se hace mejor tocando una mano, acariciando el cabello, o sencillamente, sentándose en la cama en silencio.

\section{5ㅁase: Aceptación}

Cuando el paciente ha tenido tiempo para asumir su situación y se le ha ayudado a pasar por las fases antes descritas llegará una fase en la que su destino no le deprimirá ni le enojará. Se sentirá cansado, y débil o sentirá la necesidad de dormitar a menudo. No hay que confundir esta fase con una fase feliz. Para el paciente, esta fase está desprovista de sentimientos y es la familia quien necesita más apoyo. El paciente lo único que necesita es la presencia de alguien a su lado, aunque no haya comunicación oral, simplemente el silencio, el contacto entre las manos pueden ser las comunicaciones más llenas de sentido.

Tenemos que saber que las fases en el proceso del enfermo terminal no son hieráticas, sino un proceso dinámico y por tanto no tenemos que obligar a nadie a pasar por todas y cada una de ellas, según la persona puede pasar de una fase a otra saltándose alguna de ellas o no contemplarlas. Lo que si conocemos es que el pase por estas fases facilita la aceptación de la muerte como algo natural y hace de este proceso algo idóneo, para aceptar nuestro fin, así como ayuda a la recuperación del duelo en la familia.

Durante todas estas fases el único sentimiento común que siempre persiste es la esperanza. Aún los enfermos más realistas, y los que aceptan de mejor manera su situación, mantienen una chispa de esperanza para su curación o para la aparición de un medicamento nuevo. Esta chispa de esperanza les sostiene durante días, semanas o incluso meses de sufrimiento. El papel del D. U. E. en este caso, no se trata de decirles mentiras, pero es importante compartir con el paciente su esperanza. La reacción de la familia ante la enfermedad del paciente contribuirá en mucho a la respuesta de éste. Es muy importante para el enfermo y la familia, ver que la enfermedad no rompe totalmente un hogar, ni priva completamente a todos los miembros de cualquier actividad placentera.

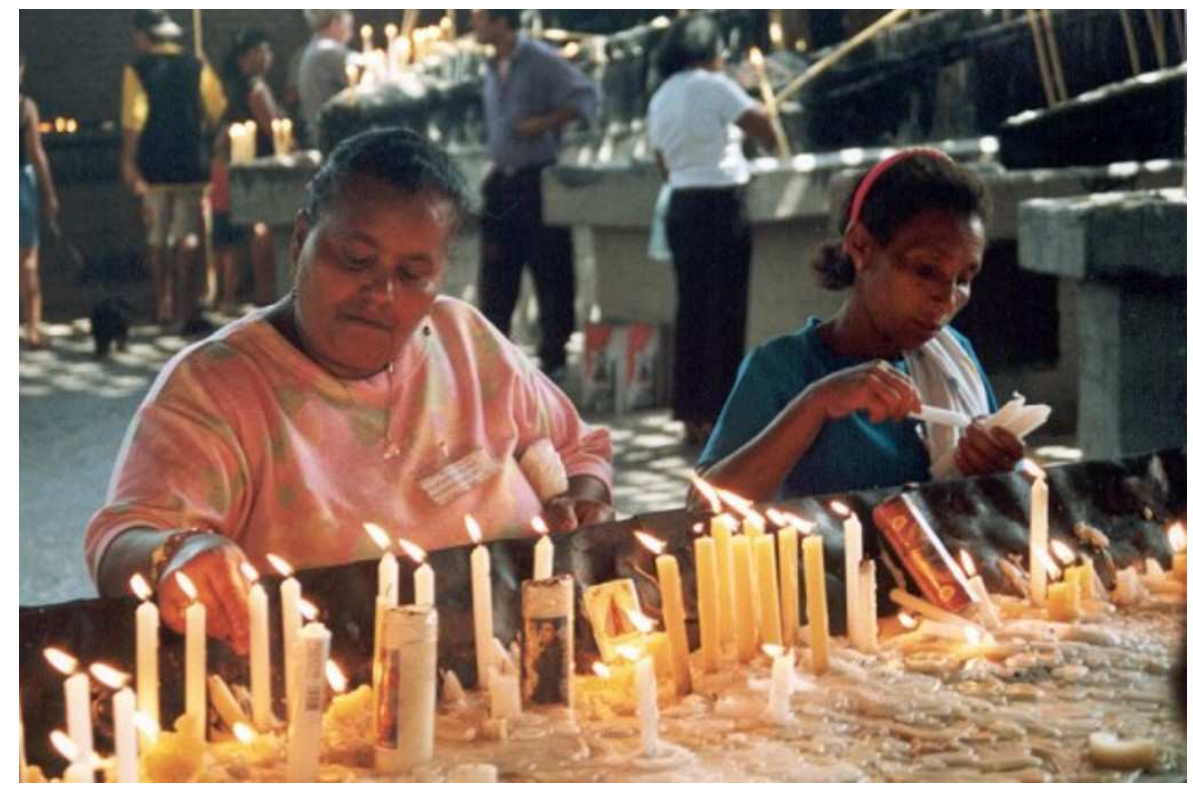

3.- El único sentimiento común que siempre persiste es la esperanza. 


\section{III.- MEDIDAS TERAPÉUTICAS EN EL PACIENTE TERMINAL.}

En los últimos años se ha investigado mucho en todo lo referente a la muerte y el morir, y se ha tratado de hallar nuevas y mejores formas de asistir a los pacientes terminales, que sería imposible enunciar aquí. La situación de enfermedad terminal, supone un verdadero reto para los D. U. E. (5) (6) por la dificultad en el manejo de una situación compleja, en la que la necesidad acuciante de controlar los síntomas no debe enturbiar la correcta valoración diagnóstica y el adecuado enfoque terapéutico.

El dolor, aun a pesar de no ser el único síntoma, sí es el que más angustia genera al paciente y a su familia. Y por ello, es prioritario abordarlo de una forma eficaz y precoz.

Debemos valorar cuidadosamente la agresividad en las decisiones terapéuticas y el beneficio esperado de las mismas. Debemos comprender que unas horas de dolor en un paciente terminal suponen un gran sufrimiento, añadido a la propia situación de terminalidad. La primera condición, por tanto, será determinar que nos encontramos ante una enfermedad en fase terminal, lo que en ocasiones no es tan fácil como pueda parecer.

En la situación de terminalidad los cambios son frecuentes en cuanto a la cualidad e intensidad de los síntomas, entre ellos el dolor, según avanza la enfermedad. Esto obliga a una revisión constante de las estrategias terapéuticas, adaptándolas a las diferentes necesidades del paciente. Lo que inicialmente puede significar la adición de medidas terapéuticas agresivas $y$, finalmente, retirar cualquier otra medicación que no vaya encaminada al control del dolor, hasta llegar a la sedación terminal si fuera preciso.

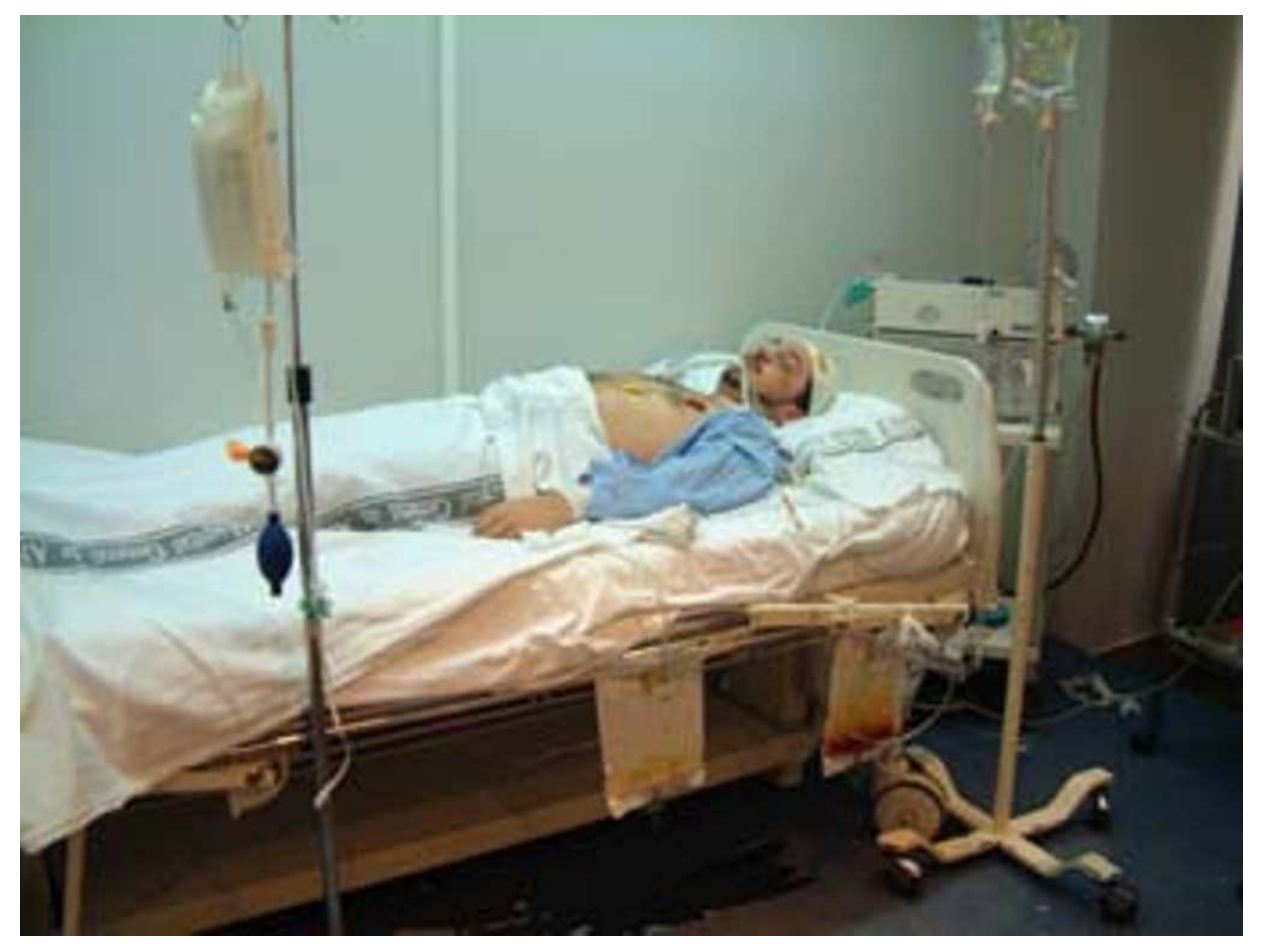

4.- Paciente en estado terminal.

El paciente desahuciado tiene necesidades muy especiales que pueden cubrirse si nos tomamos tiempo para escuchar y averiguar cuáles son. Muchos pacientes se aferran a la vida porque tienen asuntos pendientes. Todos estos se encuentran mejor después de hacer confesiones o de encontrar soluciones para el cuidado de otros, y generalmente, morían poco después de que desapareciera el asunto pendiente. 
Hay un momento en la vida de un paciente en que deja de haber dolor, y la mente deja de imaginar cosas, la necesidad de alimento se vuelve mínima y la conciencia de lo que le rodea desaparece en la oscuridad. Es entonces cuando los familiares recorren los pasillos del hospital, atormentados por la espera. En esos momentos es demasiado tarde para las palabras, y no obstante, es cuando la familia pide más ayuda con o sin palabras.

El personal sanitario puede ser una gran ayuda, durante estos momentos finales, si logran comprender los conflictos de la familia en ese momento y ayudan a seleccionar la persona que se sienta más capaz de estar junto al paciente moribundo.

\section{IV.- RECOMENDACIONES.}

A continuación expondremos algunas recomendaciones sobre cómo actuar en la relación y los cuidados al paciente terminal sacadas de la bibliografía consultada (2) (7) (8), pero previamente partimos de una idea fundamental y motivadora: "las actitudes y conductas del personal sanitario tienen consecuencias directas para el enfermo". Este principio de partida justifica la necesidad de que asimilemos correctamente estos criterios:

1. Asumir que la profesión de Enfermería brinda a sus profesionales la oportunidad especial de ayudar y confortar al moribundo, así como de proporcionar consuelo y comprensión a sus familiares, en un momento muy difícil y de acuciante necesidad de apoyo humano.

2. Que el Equipo de Salud se proporcione respaldo y comprensión mutuamente, ya que el asumir los profesionales de la salud (y especialmente los de Enfermería) la responsabilidad de un proceso terminal puede resultar sumamente estresante. Este "espíritu de equipo" incidirá directamente en la calidad de los cuidados al paciente terminal, optimizando por ello la satisfacción de sus necesidades.

3. Animar al Equipo de Salud a escuchar (y a parecer siempre dispuesto a hacerlo) a los pacientes que deseen hablar de sus miedos y temores. Procurar una relación franca y sincera entre el Equipo de Salud y el paciente y sus familiares.

4. No ser demasiado estrictos con las normas y protocolos de la institución donde trabajemos a la hora de aplicarlos, sino mas bien adaptarlos a las necesidades de cada enfermo. Así evitaremos la pérdida de autocontrol en el paciente y los consiguientes efectos estresantes, depresivos y de indefensión.

5. En cuanto a dar información al paciente sobre el pronóstico de su enfermedad, no podemos negar al enfermo tanto el derecho de conocer la verdad como de no querer conocerla. Así pues, habrá que valorar si lo desea y es conveniente o no, por medio del trato directo con él, a través de sus preguntas, actitudes y también valorándolo con la familia. No se puede decir ni negar la verdad por sistema.

6. Agilizar si estuviera hospitalizado, si el paciente y/o familia lo desean y resulta conveniente, el alta voluntaria. De esta manera el paciente puede morir en su hogar, en un ambiente íntimo rodeado de sus familiares y amigos.

7. No extremar las medidas terapéuticas para alargar la vida a costa de aumentar el sufrimiento del paciente o alejarlo del contacto humano. Quizá, así, en lugar de alargar la vida se está alargando la muerte.

8. Tener unos criterios útiles para el uso y administración de fármacos, a fin de adecuarlos a la situación de enfermedad terminal y a su objetivo de confort, teniendo en cuenta la dificultad de administración.

9. Ofrecerle al paciente la posibilidad de ayuda religiosa en las horas finales de su vida, si así lo desea.

10. Ayudar y orientar a los familiares en las formalidades postmortem: cumplimentación de impresos, derivación a otros profesionales (trabajador social, sacerdote, etc.). 
11. Trabajar con profesionalidad: si personalmente no deseamos plantearnos el tema, al menos como algo cotidiano en nuestro trabajo debemos aceptar que el fin de toda vida es la muerte. Esto, por supuesto, sin llegar a la frivolidad que se observa en muchos profesionales, que parecen estar tan acostumbrados que no personalizan la situación, comprendiendo el sufrimiento tanto del enfermo como de la familia.

12. Por último, contemplamos la asistencia a la familia en el duelo, intentando en la medida de lo posible evitar la aparición del duelo patológico.

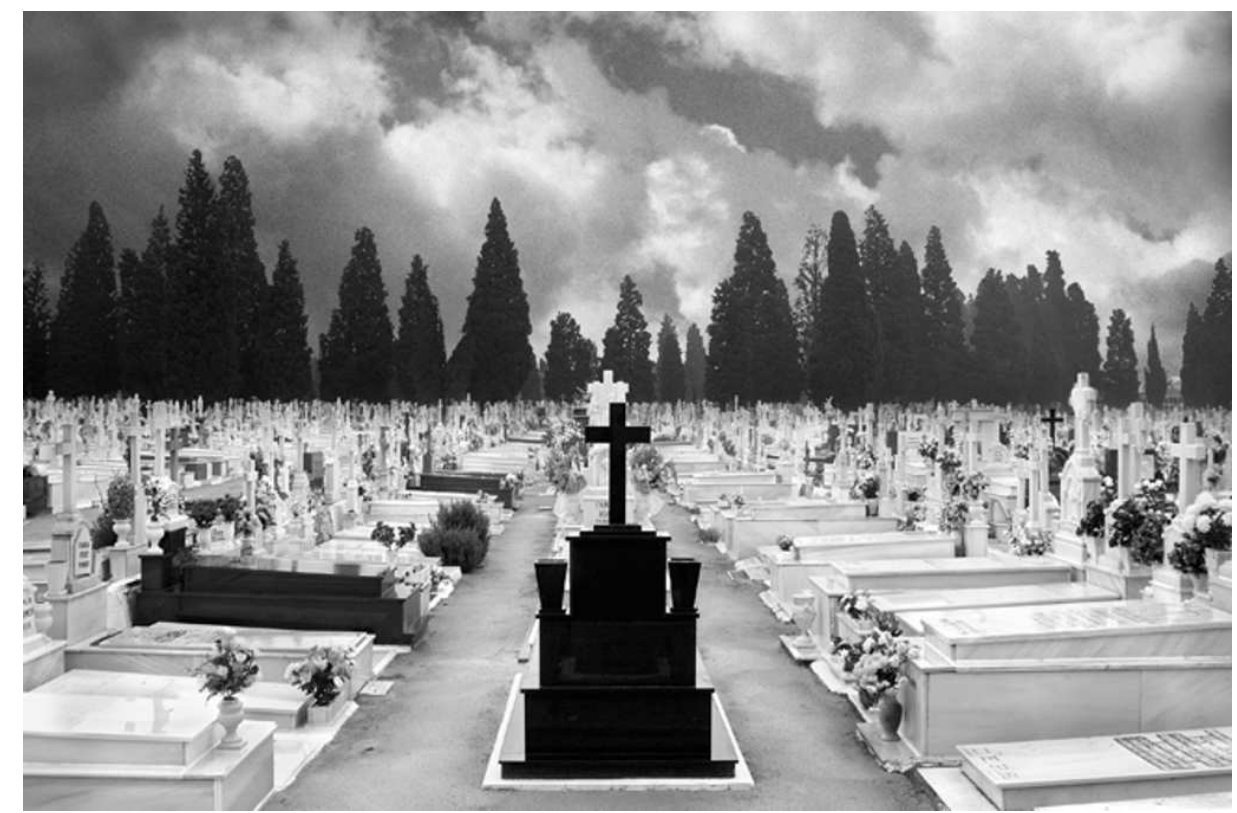

5.- Tras la muerte se debe orientar a los familiares en las formalidades postmortem

\section{V.- REFERENCIAS BIBLIOGRÁFÍCAS}

1. Cuidados Paliativos. Recomendaciones de la Sociedad Española de Cuidados Paliativos. Ministerio de Sanidad y consumo. Madrid, 1993

2. Cuidados Paliativos. Proceso Asistencial integrado. Consejería de Salud Junta de Andalucía. 2002

3. M. A. Benitez del Rosario, A. Salinas Martín. Cuidados Paliativos y Atención Primaria, aspectos de Organización. Springer - Verlag Ibérica. Barcelona. 2000

4. Astudillo W, Mendinueta C, Astudillo E. Cuidados del enfermo en fase terminal y atención a su familia. 4a . Pamplona: EUNSA; 2002.

5. Eulalia López Inmedio. Enfermería en cuidados paliativos. Panamericana. Madrid 1998.

6. Shirley E. Otto. Enfermería Oncológica. Harcourt - Brace Mosby. Madrid. 2002

7. Martín Zurro A, Cano Pérez J. F. Atención Primaria. Conceptos, organización y práctica clínica. Barcelona: Doyma, 1994

8. Cuidados Paliativos Domiciliarios. Atención integral al paciente y su familia. Consejería de Salud Junta de Andalucía. 2003.

ISSN 1695-6141

๑ COPYRIGHT Servicio de Publicaciones - Universidad de Murcia 